CERN-TH/96-57

ITP-Budapest 517

\title{
The SU(2)-Higgs model on asymmetric lattices
}

\author{
F. Csikor \\ Institute for Theoretical Physics, Eötvös University, \\ H-1088 Budapest, Hungary \\ Z. Fodor* \\ Theory Division, CERN, \\ CH-1211 Geneva 23, Switzerland
}

\begin{abstract}
We calculate the $\mathcal{O}\left(g^{2}, \lambda\right)$ corrections to the coupling anisotropies of the $\mathrm{SU}(2)$-Higgs model on lattices with asymmetric lattice spacings. These corrections are obtained by a one-loop calculation requiring the rotational invariance of the gauge- and Higgs-boson propagators in the continuum limit.
\end{abstract}

CERN-TH/96-57

February, 1996

*On leave from Institute for Theoretical Physics, Eötvös University, H-1088 Budapest, Hungary 


\section{Introduction}

At high temperatures the electroweak symmetry is restored. Since the baryon violating processes are unsuppressed at high temperatures, the observed baryon asymmetry of the universe has finally been determined at the electroweak phase transition [1].

In recent years quantitative studies of the electroweak phase transition have been carried out by

means of resummed perturbation theory [2]- [4] and lattice Monte Carlo simulations [5]- 13]. In the SU(2)-Higgs model for Higgs masses $\left(M_{H}\right)$ below $50 \mathrm{GeV}$, the phase transition is predicted by the perturbation theory to be of first order. However, no definite statement can be made for physically more interesting masses, e.g. $M_{H}>80 \mathrm{GeV}$. Due to the bad infrared features of the theory, the perturbative approach breaks down in this parameter region. A systematic and fully controllable treatment is necessary, which can be achieved by lattice simulations.

For smaller Higgs boson masses $\left(M_{H}<50 \mathrm{GeV}\right)$ the phase transition is quite strong and relatively easy to study on the lattice. For larger $M_{H}$ (e.g. $M_{H}=80 \mathrm{GeV}$ ) the phase transition gets weaker, the lowest excitations have masses small compared to the temperature, $T$. From this feature one expects that a finite temperature simulation on isotropic lattice would need several hundred lattice points in the spatial directions even for $L_{t}=2$ temporal extension. These kinds of lattice sizes are out of the scope of the present numerical resources.

One possibility to solve the problem of these different scales is to integrate out the heavy, $\mathcal{O}(T)$ modes perturbatively, and analyse the obtained theory on the lattice. This strategy turned out to be quite successful, and both its perturbative and lattice features have been studied by several groups [6]- [11.

With this paper we follow another approach (analytic and in the future Monte Carlo) to handle this two-scale problem. We will use the simple idea that finite temperature field theory can be conveniently studied on asymmetric lattices, i.e. lattices with different spacings in temporal $\left(a_{t}\right)$ and spatial $\left(a_{s}\right)$ directions. This method solves the two-scale problem in a natural way [14]. Another advantage is, well-known and often used in QCD, that this formulation makes an independent variation of the temperature $(T)$ and volume $(V)$ possible. The perturbative corrections to the coupling anisotropies are known in QCD (see refs. [15, 16]).

The plan of this letter is as follows. In Section 2 we give the lattice action of the model on asymmetric lattices and discuss the effective potential. Section 3 contains the calculation of the wave function quantum correction terms, which give the quantum corrections to the anisotropy parameters. Section 4 is devoted to the discussions and outlook.

\section{Lattice action and the critical hopping parameter}

For simplicity, we use equal lattice spacings in the three spatial directions $\left(a_{i}=a_{s}, i=1,2,3\right)$ and another spacing in the temporal direction $\left(a_{4}=a_{t}\right)$. The asymmetry of the lattice spacings is characterized by the asymmetry factor $\xi=a_{s} / a_{t}$. The different lattice spacings can be ensured by different coupling strengths in the action for time-like and space-like directions. The action reads

$$
\begin{aligned}
S[U, \varphi]= & \beta_{s} \sum_{s p}\left(1-\frac{1}{2} \operatorname{Tr} U_{p l}\right)+\beta_{t} \sum_{t p}\left(1-\frac{1}{2} \operatorname{Tr} U_{p l}\right) \\
& +\sum_{x}\left\{\frac{1}{2} \operatorname{Tr}\left(\varphi_{x}^{+} \varphi_{x}\right)+\lambda\left[\frac{1}{2} \operatorname{Tr}\left(\varphi_{x}^{+} \varphi_{x}\right)-1\right]^{2}\right. \\
& \left.-\kappa_{s} \sum_{\mu=1}^{3} \operatorname{Tr}\left(\varphi_{x+\hat{\mu}}^{+} U_{x, \mu} \varphi_{x}\right)-\kappa_{t} \operatorname{Tr}\left(\varphi_{x+\hat{4}}^{+} U_{x, 4} \varphi_{x}\right)\right\},
\end{aligned}
$$



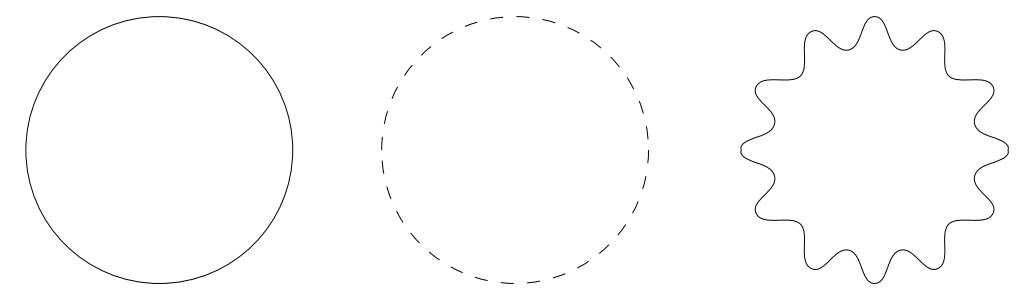

Figure 1: One-loop diagrams contributing to the effective potential. The solid line represents the Higgs-, the dashed one the Goldstone- and the wavy line the vector-boson.

where $U_{x, \mu}$ denotes the $\mathrm{SU}(2)$ gauge link variable, $U_{s p}$ and $U_{t p}$ the path-ordered product of the four $U_{x, \mu}$ around a space-space or space-time plaquette, respectively. The symbol $\varphi_{x}$ stands for the Higgs field, which is also written as $\varphi_{x}=\rho_{x} \cdot \alpha_{x}$, with $\rho_{x} \in \mathbf{R}^{+}$and $\alpha_{x} \in \mathrm{SU}(2)$.

The anisotropies

$$
\gamma_{\beta}^{2}=\frac{\beta_{t}}{\beta_{s}} \quad, \quad \gamma_{\kappa}^{2}=\frac{\kappa_{t}}{\kappa_{s}}
$$

are functions of the asymmetry $\xi$. On the tree-level the coupling anisotropies are equal to the lattice spacing asymmetry; however, they receive quantum corrections in higher orders of the loop-expansion

$$
\gamma_{\beta}^{2}=\xi^{2}\left[1+c_{\beta}(\xi) g^{2}+b_{\beta}(\xi) \lambda+\mathcal{O}\left(g^{4}, \lambda^{2}\right)\right], \quad \gamma_{\kappa}^{2}=\xi^{2}\left[1+c_{\kappa}(\xi) g^{2}+b_{\kappa}(\xi) \lambda+\mathcal{O}\left(g^{4}, \lambda^{2}\right)\right] .
$$

Here $g$ is the bare gauge coupling in standard notation and a formal double expansion in $g^{2}$ and $\lambda$ has been performed. In this double expansion we use the formal power counting $\lambda \sim g^{2}$. It is useful to introduce the hopping parameter $\kappa^{2}=\kappa_{s} \kappa_{t}$ and $\beta^{2}=\beta_{s} \beta_{t}$. In general, the determination of $\gamma_{\beta}(\xi)$ and $\gamma_{\kappa}(\xi)$ should be done non-perturbatively. This can be achieved by requiring that the Higgs- and W-boson correlation lengths in physical units are the same in the different directions. This idea can be applied in perturbation theory as well (see e.g. [16]), and we will follow this method in our analysis too. It is believed that this procedure ensures a rotationally invariant effective action [16, 17].

The Feynman-rules of the theory can be derived as usual (for the three-dimensional case they can be found in ref. [7]). In our analysis we will need the Higgs- and gauge-boson propagators with one-loop corrections. The inverse of the tree-level Higgs-boson propagator has the form

$$
\Delta_{H, 0}(p)^{-1}=m_{H, 0}^{2}+\sum_{i=1}^{3} \hat{p}_{i}^{2}+\frac{\gamma_{\kappa}^{2}}{\xi^{2}} \hat{p}_{4}^{2}
$$

for the gauge-boson:

$$
\Delta_{W, 0, \mu \nu}^{a b}(p)^{-1}=\delta^{a b} \delta_{\mu \nu}\left[m_{W, 0}^{2}+\sum_{i=1}^{3} \hat{p}_{i}^{2}+\frac{\gamma_{\beta}^{2}}{\xi^{2}} \hat{p}_{4}^{2}\right] .
$$

Here Feynman-gauge has been used in the Higgs (broken) phase and

$$
\hat{p}_{i}=\frac{2}{a_{s}} \sin \frac{a_{s} p_{i}}{2}, \quad \hat{p}_{4}=\frac{2}{a_{t}} \sin \frac{a_{t} p_{4}}{2} .
$$

The tree-level masses are given by

$$
m_{H, 0}^{2}=-\frac{2}{a_{s}^{2}}\left[\frac{1-2 \lambda}{\kappa} \xi-6-2 \xi^{2}\right], \quad m_{W, 0}^{2}=\frac{m_{H, 0}^{2} \kappa^{2}}{2 \lambda \xi \beta} .
$$




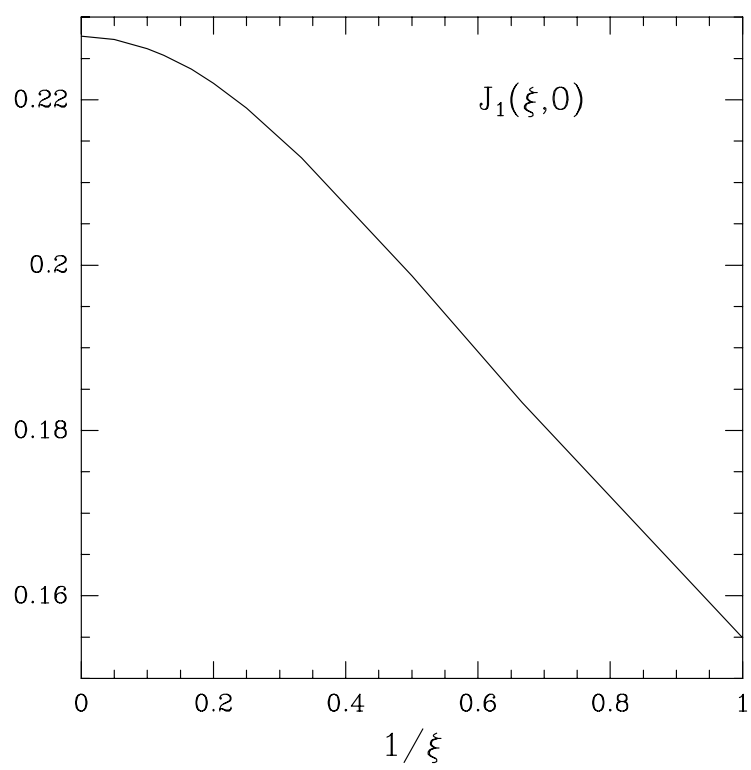

Figure 2: The lattice integral $J_{1}(\xi, 0)$ (see text) on asymmetric lattices as a function of $1 / \xi$.

Lattice perturbation theory is conveniently formulated by means of the parameters $\lambda_{c}$ and $g$

$$
\lambda_{c}=\frac{\lambda \xi}{4 \kappa^{2}}, \quad g^{2}=\frac{4}{\beta}
$$

The main goal of the paper is to perform a one-loop analysis of the theory defined by eq. (11). This means first the determination of the mass-counterterm. One wants to tune the bare parameters in a way that the one-loop renormalized masses are finite in the continuum limit (however, their values in lattice units vanish $a_{s} M_{r e n}=0$ for $a_{s} \rightarrow 0$ ). At the same time the vacuum expectation value of the scalar field will be also zero in lattice units $\left(a_{s} v=0\right.$ for $\left.a \rightarrow 0\right)$, i.e. we are at the phase transition point between the spontaneously broken Higgs phase and the $\mathrm{SU}(2)$ symmetric phase. The condition is fulfilled by an appropriate choice of the hopping parameter (critical hopping parameter). The ratios of the couplings $\left(\gamma_{\beta}\right.$ and $\left.\gamma_{\kappa}\right)$ are still free parameters and can be fixed by two additional conditions. We demand rotational (Lorenz) invariance for the scalar and vector propagators on the one-loop level. This ensures that the propagators with one-loop corrections have the same form in the $z$ and $t$ directions. Clearly, arbitrary couplings for different directions in eq. (1) would not lead to such rotationally invariant two-point functions.

The most straightforward method to determine the transition point is the use of the effective potential in the Landau gauge. The relevant one-loop graphs are shown in Figure 1. In this gauge no contribution comes from the ghost fields. The direct calculation gives for a constant $\Phi$ field

$$
V_{e f f}(\Phi)=-\frac{m_{H, 0}^{2}}{4} \Phi^{2}+\lambda_{c} \Phi^{4}+\int_{k}\left[\frac{1}{2} \log \left(\hat{k}^{2}+12 \lambda_{c} \Phi^{2}\right)+\frac{3}{2} \log \left(\hat{k}^{2}+4 \lambda_{c} \Phi^{2}\right)+\frac{9}{2} \log \left(\hat{k}^{2}+g^{2} \Phi^{2} / 4\right)\right]
$$

where

$$
\int_{k} \equiv \frac{1}{(2 \pi)^{4}} \int_{-\pi / a_{s}}^{\pi / a_{s}} d^{3} k \int_{-\pi / a_{t}}^{\pi / a_{t}} d k_{4}
$$

and

$$
\hat{k}^{2} \equiv \sum_{\mu=1}^{4} \hat{k}_{\mu}^{2} .
$$



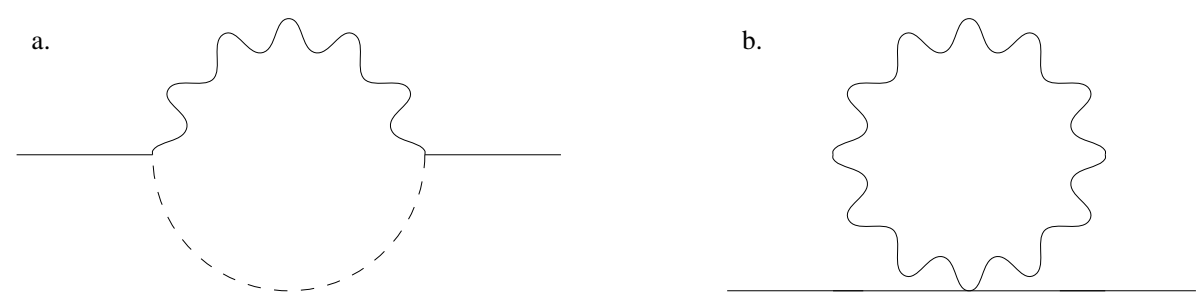

Figure 3: Self-energy graphs of the Higgs-boson contributing to the anisotropy parameters.

Note that, in this order, the propagators have no $\gamma_{\kappa, \beta}^{2} / \xi^{2}$-type corrections in the temporal direction. These terms would lead to corrections of higher order in the critical hopping parameter. The condition $d^{2} V_{e f f}(\Phi=0) / d \Phi^{2}=0$ gives the value of the critical hopping parameter

$$
\kappa_{c}=\frac{\xi}{2\left(3+\xi^{2}\right)}+\frac{1}{\left(3+\xi^{2}\right)^{2}}\left[6 \xi J_{1}(\xi, 0)-\frac{\xi^{2}}{\left(3+\xi^{2}\right)}\right] \lambda_{c}+\frac{9 \xi J_{1}(\xi, 0)}{16\left(3+\xi^{2}\right)^{2}} g^{2},
$$

where the notation

$$
J_{n}\left(\xi, m a_{s}\right)=a_{s}^{4-2 n} \int_{k} \frac{1}{\left(m^{2}+\hat{k}^{2}\right)^{n}}
$$

for the dimensionless lattice integrals on asymmetric lattices is used. For the readers' convenience we plot $J_{1}(\xi, 0)$ of eq. (12) in Figure 2 as a function of $1 / \xi$. We have proved that eq. (12) does not depend on the choice of the gauge parameter in $R_{\xi}$ gauge. For the special case of symmetric lattice spacings, $\xi=1$, our quantum corrections to the critical hopping parameter reproduce the known result of the isotropic SU(2)-Higgs model [17].

\section{Quantum corrections to the anisotropy parameters}

On the one-loop level the propagators of eqs. (田 and 5) receive quantum corrections:

$$
\Delta_{H, 1}(p)^{-1}=\Delta_{H, 0}(p)^{-1}+\Sigma_{H, 1}(p), \quad \Delta_{W, 1, \mu \nu}^{a b}(p)^{-1}=\Delta_{W, 0, \mu \nu}^{a b}(p)^{-1}+\Sigma_{W, 1, \mu \nu}^{a b}(p) .
$$

One can demand rotational invariance in the continuum limit, $a_{s}, a_{t} \rightarrow 0$ at fixed $\xi=a_{s} / a_{t}$. The corrections to the anisotropies in the kinetic parts of eqs. (4,5) should be cancelled by the kinetic parts of the self-energies (14) (cf. [16]). For the Higgs-boson this can be achieved by demanding

$$
\frac{\gamma_{\kappa}^{2}}{\xi^{2}}+\left.\frac{1}{2} \frac{\partial^{2} \Sigma_{H, 1}(p)}{\partial p_{4}^{2}}\right|_{p=0}=1+\left.\frac{1}{2} \frac{\partial^{2} \Sigma_{H, 1}(p)}{\partial p_{i}^{2}}\right|_{p=0}
$$

where $i=1,2,3$. An analogous condition can be given for the gauge-boson self-energy as well. These conditions determine the functions $c_{\beta}(\xi), c_{\kappa}(\xi), b_{\beta}(\xi)$ and $b_{\kappa}(\xi)$ of eq. (3).

In this section Feynman-gauge will be used. The relevant graphs to eq. (15) are shown in Figure 3. The diagrams contributing to the analogous condition in the gauge sector are given by Figure 4 . Note that there are several other one-loop contributions to the self-energies; however, those graphs give higher order terms in the $g^{2}, \lambda$ double expansion, or they are independent of the external momenta.

The contribution due to the Higgs $\rightarrow$ Goldstone+vector $\rightarrow$ Higgs graph reads

$$
\frac{3 g^{2}}{4} \int_{k} \frac{(\widehat{p+k})^{2}}{\left(\hat{k}^{2}+M_{W}^{2}\right)\left((\widehat{p-k})^{2}+M_{W}^{2}\right)}
$$



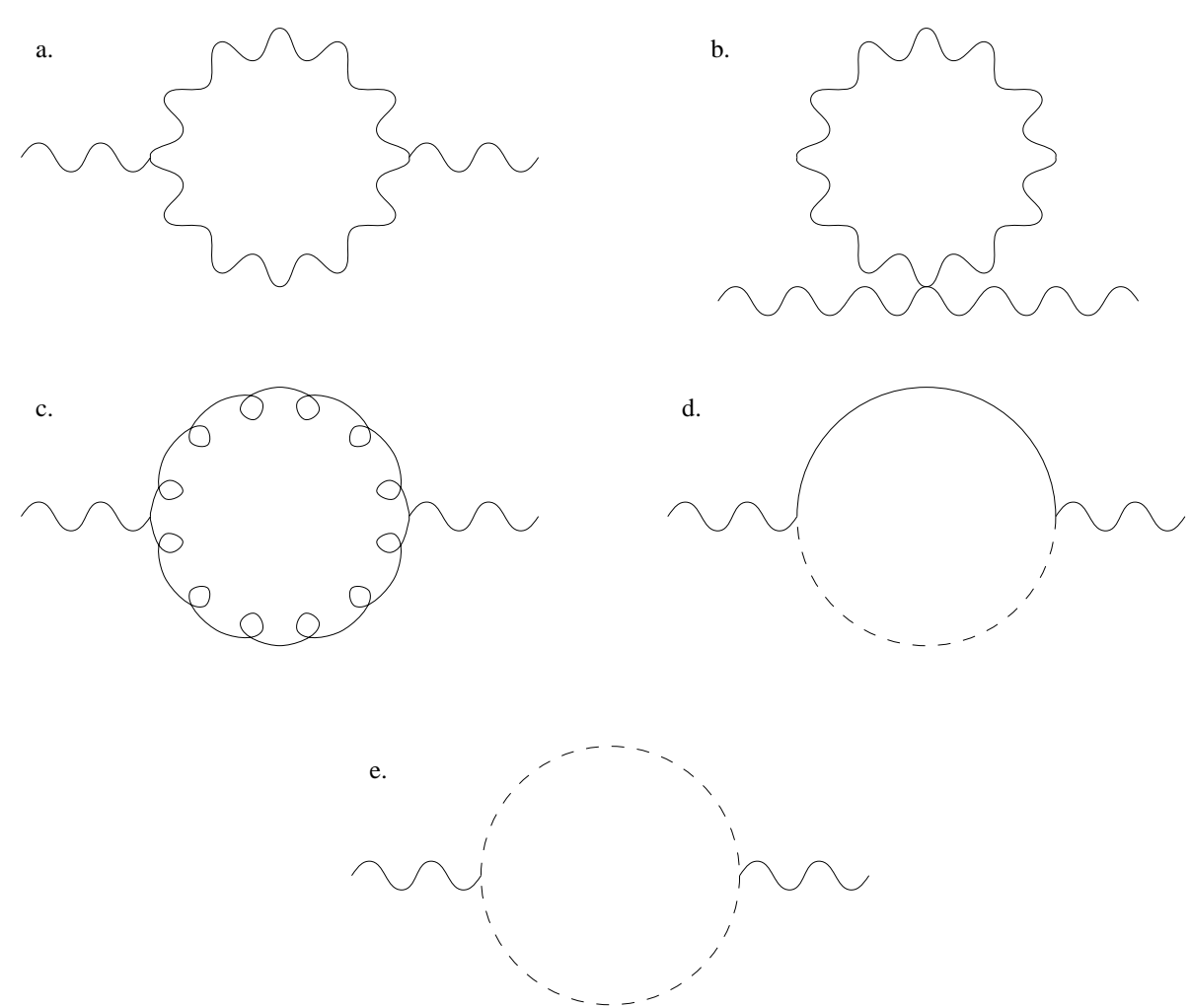

Figure 4: Self-energy graphs of the vector-boson contributing to the anisotropy parameters. The curly line represents the ghost field.

whereas the Higgs $\rightarrow$ vector $\rightarrow$ Higgs with four-particle vertex gives

$$
-\frac{3 g^{2}}{4} \int_{k} \sum_{\mu=1}^{4} \cos ^{2} p_{\mu} a_{\mu} \frac{1}{\hat{k}^{2}+M_{W}^{2}} .
$$

The contributions to the vector self-energy are much more complicated (particularly the one due to the vector four-coupling of Figure 4.b); therefore, they will be not listed here. The corrections to the anisotropies are obtained as a difference of two integrals -see eq. (15)- which is always finite. The functions $c_{\beta}(\xi)$ and $c_{\kappa}(\xi)$ of eq. (3) are plotted in Figure 5.

There are several important features of the result, which should be mentioned. a. Masses in the propagators: a consistent perturbative procedure on the lattice determines the bare parameters, for which the renormalized masses vanish, cf. eq. (12). With these bare couplings other quantities, e.g. asymmetry parameters, are determined. However, using the one-loop renormalized masses $\left(a_{s} M_{H}=a_{s} M_{W}=0\right)$ in the propagators instead of the bare ones leads to changes in the results, which are higher order in $g^{2}$ and $\lambda$. Therefore, all our results are given by the integrals with renormalized masses.

b. $g^{2}$ and $\lambda$ corrections: In Figure 5 we have given only $c_{\beta}(\xi)$ and $c_{\kappa}(\xi)$. The functions $b_{\beta}(\xi)$ and $b_{\kappa}(\xi)$ vanish, thus there are no corrections of $\mathcal{O}(\lambda)$ to the anisotropy parameters. It is easy to understand this result qualitatively, since only graphs with two or more scalar self-interaction vertices have nontrivial dependence on the external momentum. This feature is connected with the well-known fact that the $\Phi^{4}$ theory does not have any wave function correction in first order in the scalar self-coupling. It is worth mentioning that there is only one type of two-loop graph (the setting-sun) which should be combined with the one-loop graphs, in order to obtain the whole $\mathcal{O}\left(\lambda^{2}\right)$ correction.

c. Pure gauge theory: The graphs of Figure 4.a, 4.b and 4.c are identical with those of the pure gauge 


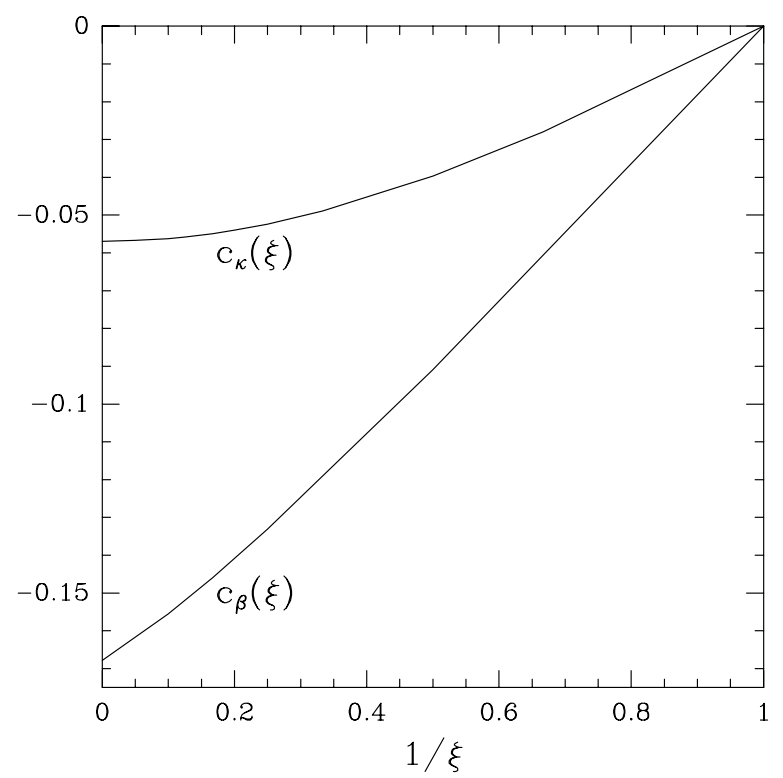

Figure 5: $\quad c_{\beta}(\xi)$ and $c_{\kappa}(\xi)$ as functions of $1 / \xi$.

theory. Evaluating these diagrams one reproduces the result of ref. [8] (the function $c_{\beta}(\xi)$ of the present paper corresponds to $c_{\tau}(\xi)-c_{\sigma}(\xi)$ of ref. [8]). The most important contribution comes from the self-energy graph with vector four-coupling of Figure 4.b. Inclusion of the scalar particles gives only small changes. The relative difference between the $c_{\beta}(\xi)$ functions for the pure $\mathrm{SU}(2)$ theory and for the SU(2)-Higgs model is typically a few $\%$.

d. Quantum corrections to the hopping parameter: the contributions to the hopping parameter come from Figure 3.a and 3.b. This correction has the same sign and order of magnitude than that of the gauge anisotropy parameter; however it is somewhat smaller. It is possible to combine the anisotropies $c_{\beta}^{\prime}(\xi)=c_{\beta}(\xi)-c_{\kappa}(\xi)$. For this choice in the gauge sector and with $\gamma_{\kappa}=\xi$ the rotational invariance can be restored on the one-loop level, choosing the appropriate value for the lattice spacing asymmetry $a_{s} / a_{t}$. Thus, the masses in both directions will be the same. However, the obtained lattice spacing asymmetry will then slightly differ from the original $\xi$; one gets $a_{s} / a_{t}=\xi\left(1-g^{2} c_{\kappa}(\xi) / 2\right)+\mathcal{O}\left(g^{4}, \lambda^{2}\right)$.

Finally, it should be emphasized that the truly non-perturbative analysis of several thermodynamical quantities on lattices with anisotropic couplings ought to include the non-perturbative determination of the anisotropy parameters. This can be achieved by the study of the correlation functions and/or static potentials given by Wilson-loops.

\section{Discussion}

We have studied the $\mathrm{SU}(2)$-Higgs model on lattices with asymmetric lattice spacings $a_{s} \neq a_{t}$. We have determined the $\mathcal{O}\left(g^{2}, \lambda\right)$ corrections to the coupling anisotropy parameters $\gamma_{\beta}$ and $\gamma_{\kappa}$. The corrections of order $\lambda$ vanish. The $g^{2}$ corrections are quite small. Since the finite temperature electroweak phase transition is characterized by the dimensionless gauge-coupling $g^{2} \approx 0.5$, the one-loop perturbative approach to the lattice anisotropy parameters seems to be satisfactory.

Special thanks go to I. Montvay for essential proposals. Discussions with K. Kajantie, F. Karsch, A. Patkós, M. Shaposhnikov and R. Sommer are also acknowledged. This work was partially supported by Hungarian Science Foundation grant under Contract No. OTKA-T016248/7. 


\section{References}

[1] V. A. Kuzmin, V. A. Rubakov, M. E. Shaposhnikov, Phys. Lett. B155 (1985) 36.

[2] P. Arnold, O. Espinosa, Phys. Rev. D47 (1993) 3546; W. Buchmüller, Z. Fodor, T. Helbig , D. Walliser, Ann. Phys. 234 (1994) 260.

[3] Z. Fodor, A. Hebecker, Nucl. Phys. B432 (1994) 127.

[4] W. Buchmüller, Z. Fodor, A. Hebecker, Nucl. Phys. B447 (1995) 317.

[5] B. Bunk, E.-M. Ilgenfritz, J. Kripfganz , A. Schiller, Nucl. Phys. B403 (1993) 453.

[6] K. Farakos, K. Kajantie, K. Rummukainen, M. Shaposhnikov, Nucl. Phys. B407 (1993) 356; B425 (1994) 67; B442 (1995) 317; K. Kajantie, M. Laine, K. Rummukainen, M. Shaposhnikov, hep-lat/9510020.

[7] M. Laine, Nucl. Phys. B451 (1995) 484.

[8] F. Karsch, T. Neuhaus, A. Patkós, Nucl. Phys. B441 (1995) 629.

[9] A. Jakovác, K. Kajantie, A. Patkós, Phys. Rev. D49 (1994) 6810; A. Jakovác, A. Patkós, P. Petreczky, hep-ph/9510230.

[10] H.-G. Dosch, J. Kripfganz, A. Laser, M. G. Schmidt, Phys. Lett. B365 (1995) 213; E. M. Ilgenfritz, J. Kripfganz, H. Perlt, A. Schiller, Phys. Lett. B356 (1995) 561.

[11] W. Buchmüller, O. Philipsen, Nucl. Phys. B443 (1995) 47; O. Philipsen, M. Teper, H. Wittig, hep-lat/9602006.

[12] F. Csikor, Z. Fodor, J. Hein, K. Jansen, A. Jaster, I. Montvay, Phys. Lett. B334 (1994) 405; Z. Fodor, J. Hein, K. Jansen, A. Jaster, I. Montvay, Nucl. Phys. B439 (1995) 147; F. Csikor, Z. Fodor, J. Hein , J. Heitger, Phys. Lett. B357 (1995) 156; F. Csikor, Z. Fodor, J. Hein, A. Jaster, I. Montvay, hep-lat/9601016.

[13] K. Jansen, hep-lat/9509018.

[14] I. Bender et al., Nucl. Phys. Proc. Suppl. 17 (1990) 387; B. Bunk, ibid 42 (1995) 566.

[15] F. Karsch, Nucl. Phys. B205 (1982) 285.

[16] F. Karsch, O. Stamatescu, Phys. Lett. B227 (1989) 153.

[17] A. Hasenfratz, P. Hasenfratz, Phys. Rev. D34 (1986) 3160; I. Montvay, Phys. Lett. B172 (1986) 71; Nucl. Phys. B293 (1987) 479. 